\title{
Evolutionary relationships in the genus Zea: analysis of repetitive sequences used as cytological FISH and GISH markers
}

\author{
Lidia Poggio ${ }^{1,2}$, Viviana Confalonieri², Cecilia Comas $^{2}$, Graciela Gonzalez' and Carlos A. Naranjo $^{1}$ \\ ${ }^{1}$ Centro de Investigaciones Genéticas (CONICET-UNLP-CIC), Instituto Fitotécnico de Santa Catalina (FCAF, UNLP) \\ C.C. 4, 1836 Llavallol, Buenos Aires, Argentina. Send correspondence to L.P. Fax: +54-11-4298-1566. \\ ${ }^{2}$ Departamento de Ciencias Biológicas (FCEN, UBA).
}

\begin{abstract}
The present study is a revision of our work on evolutionary cytogenetics of the genus Zea, including several new experiments which give a deeper insight into the nature of the DNA sequences involved in telomeric regions of Zea luxurians. These new experiments, based on the Southern blotting technique and in situ hybridization, have demonstrated the following: 1) in situ hybridization (FISH) demonstrated the presence of the 180-bp repeat maizeknob-repeat-sequence in DAPI-positive terminal heterochromatic blocks of Z. luxurians (ZL-THB region); 2) Southern blot analysis confirmed that the 180 -bp repeat present in maize is also present in Z. diploperennis, Z. luxurians and Tripsacum dactyloides, but not in Z. perennis; 3) another sequence with targeted sites for endonucleases, but without recognition sites for the 180-bp repeat, may be interspersed with the 180 -bp repeat in a tandem array sited in the ZL-THB region; 4) in situ hybridization (GISH) of probes and blocking-probes with chromosomes of Z. luxurians (using Z. luxurians as a probe and Z. diploperennis or Z. perennis as a blocking-probe) gave strong fluorescence in both cases. Since Z. diploperennis possesses the 180-bp repeat, fluorescence on Z. luxurians chromosomes was not expected. These results can be explained if the ZL-THB regions are composed not only of 180-bp repeats interspersed with other sequences, but also of other tandem arrays unique to Z. luxurians, which, according to our GISH results, are probably located at the subterminal position.
\end{abstract}

\section{INTRODUCTION}

Maize is one of the plants which has been most studied, but its origin, however, has not yet been solved. There is much data indicating that maize (Zea mays ssp. mays) with a chromosome complement of $2 n=20$ is a cryptic polyploid with an ancestral basic number of five $(x=5)$. Previous cytological findings supporting this hypothesis are: a) the existence of chromosome pairing during the meiosis of haploids (McClintock, 1933; Ting, 1985), b) the secondary association of bivalents (Vijendra Das, 1970) and c) tridimensional chromosome distribution in somatic metaphases, where the chromosomes form four groups of 5 chromosomes each (Bennett, 1983, 1984).

The genus Zea (Tribe Maydeae) is composed of two sections: the Luxuriantes Section (Doebley \& Iltis), which includes the perennials Z. diploperennis Iltis, Doebley \& Guzman and Z. perennis (Hitch.) Reeves \& Mangelsdorf and the annual Z. luxurians (Durieu \& Ascherson) Bird, and the Zea Section with the annual Z. mays L., which has been divided into subspecies (Doebley and Iltis, 1980; Iltis and Doebley, 1980). All the above mentioned species have $2 n=20$ except Z. perennis, which has $2 n=40$.

The relative affinity between the genomes in the genus Zea can be evaluated by measuring the association of the chromosomes of hybrids at diakinesis-metaphase I, a method which is particularly useful in hybrids from polyploid species because pairing occurs in the same genetic background. Since 1987 our team have been analyzing meiotic configurations in Zea species and their intra- and interespecific hybrids. These studies, discussed below, have allowed us to record cytogenetic evidence confirming the cryptic polyploid nature of the genus.

$$
\text { Meiotic behavior of } 2 n=30 \text { hybrids }
$$

The most frequent meiotic configurations in $2 n=30$ hybrids were 5 trivalents (III) +5 bivalents (II) +5 univalents (I) (Figure 1). Most of the trivalents were of the "frying pan" type, while bivalents were homomorphic even in those cases in which significant differences in chromosome size between parental species were observed (i.e., $Z$. perennis x Z. luxurians) (Molina and Naranjo, 1987; Naranjo et al., 1990; Poggio and Naranjo, 1995), and there is genetic evidence supporting the hypothesis that the univalents could have originated from the $2 \mathrm{n}=20$ species, when they occur in $2 n=30$ hybrids (Longley, 1924). There is also a tendency for bivalents to be spatially grouped; we proposed that they could belong to a $Z$. perennis genome (Molina and Naranjo, 1987; Poggio and Naranjo, 1995; Poggio et al., 1998, 1999b).

\section{Meiotic behavior of species and hybrids with $2 \mathrm{n}=20$ chromosomes}

The secondary association of bivalents was observed at diplotene-diakinesis, with a maximum of five groups of two bivalents each (Molina and Naranjo, 1987; Naranjo et al., 1990; Poggio and Naranjo, 1995), which could be evidence of association between homologous chromosomes belonging to different genomes. 


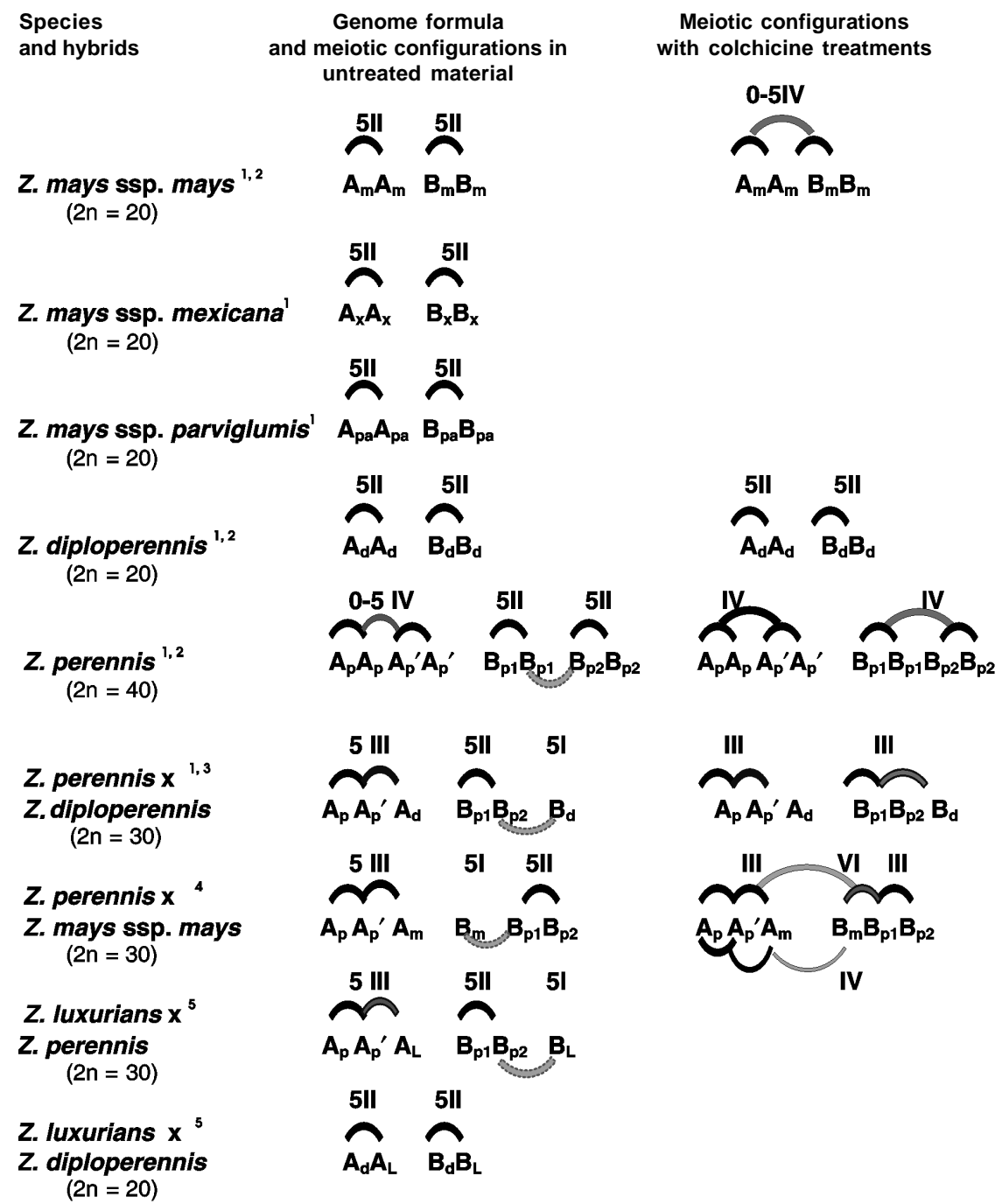

Figure 1 - Genome formulas and meiotic configurations for Zea species and hybrids with and without colchicine treatment. In untreated material: Black arcs of the top show the high-frequency meiotic association. Green arcs below indicate meiotic low frequency association. In treated material: Grey arcs indicate the new configurations that were observed and a proposed explanation for their formation. Data from: 1 = Molina and Naranjo, 1987; Naranjo et al., 1990.2 = Poggio et al., 1990. 3 = Naranjo et al., 1994. 4 = Poggio, L. and Naranjo, C.A. (unpublished results). 5 = Poggio et al., 1998; Poggio et al., 1999b.

In a variable proportion of cells, the 10 bivalents were divided into two groups of five bivalents at diplotene-metaphase I which, together with other evidence, indicates that five is the basic chromosome number $(\mathrm{x}=5)$ of the genus and suggests the separation of two relict genomes (designated as genomes A and B) (Naranjo et al., 1990; Poggio and Naranjo, 1995). In alloplasmic maize lines (composed of maize nuclei and Z. mays ssp. mexicana (teosinte) cytoplasm), the separation of bivalents into two groups was both noticeable and very frequent, suggesting that interactions between the nucleus and the cytoplasm influence the spatial separation of genomes (Poggio and Naranjo, 1995; Poggio et al., 1997).

Another important characteristic observed in allo- plasmic lines and diploid hybrids was that in about $20-50 \%$ of pollen mother cells (PMC) at prophase I, MI and AI, the two groups of bivalents, are asynchronous, showing a slight displacement in the progress of meiosis, one group of 5 bivalents being more advanced with respect to the other (Poggio et al., 1997). Since this phenomenon is common in interspecific and intrageneric hybrids (Bennett, 1983; Jouve et al., 1977) it can be assumed that in Zea the two groups of 5 bivalents each correspond to different ancestral genomes (genomes A and B), a hypothesis also supported by the presence of two nucleoli in $10 \%$ of the cells in alloplasmic lines and hybrids, each nucleoli being associated with one of the groups of 5 bivalents (Poggio et al., 1997). 
Treatment of species and hybrids with dilute colchicine

Jackson and Murray (1983) demonstrated that dilute colchicine applied at the onset of meiosis promotes intergenomic pairing and reveals cryptic homologies in amphiploids. Poggio et al. (1990) and Naranjo et al. (1994) have studied the meiotic configuration of several Zea species and hybrids treated with colchicine. These workers have found that in $Z$. mays ssp. mays $(2 \mathrm{n}=20) 1$ to 5 tetravalents are formed, demonstrating that cryptic homologies exist between the A and B genomes (described above), designated as Am and Bm for Z. mays ssp. mays (Figure 1). In Z. diploperennis $(2 \mathrm{n}=20)$ no quadrivalents were found, and no noticeable homologies between the $\mathrm{Ad}$ and $\mathrm{Bd}$ genomes were detected ( $\mathrm{Ad}$ and $\mathrm{Bd}$ corresponding to $\mathrm{Z}$. diploperennis genomes) (Figure 1$)$. In $Z$. perennis $(2 \mathrm{n}=40)$ the number of quadrivalents increased to ten, revealing some cryptic homologies within the Ap genome and more within the $\mathrm{Bp}$ genome (Ap and $\mathrm{Bp}$ corresponding to $\mathrm{Z}$. perennis genomes). The absence of octovalents demonstrated the lack of homology between Ap and Bp genomes (Figure 1). In $Z$. diploperennis $\times$ Z. perennis hybrids $(2 n=30)$ the frequency of trivalents in cells not treated with colchicine was $25 \%$ while in colchicine-treated cells it was $90 \%$, but hexavalents were not observed in this hybrid. These results show greater homologies between $Z$. perennis $\mathrm{B}$ genomes (Bp) and Z. diploperennis $\mathrm{B}$ genomes $(\mathrm{Bd})$ than those detected in untreated material (Figure 1). In the hybrid Z. mays ssp. mays $x Z$. perennis $(2 \mathrm{n}=30)$ there was an increase in trivalent frequency, and quadrivalent and hexavalent formation was observed in $30 \%$ of the cells in agreement with the homologies detected between Z. mays ssp. mays genomes $\mathrm{Am}$ and $\mathrm{Bm}$ in colchicine-treated material (Figure 1). The formation of these hexavalents constitutes one of the most striking pieces of evidence supporting the hypothesis that maize is a cryptic tetraploid while $Z$. perennis is a cryptic octoploid (more details to be published later by Poggio and Naranjo).

Poggio et al. (1990) and Naranjo et al. (1994) showed that in species and hybrids of the genus Zea, colchicine treatment may disrupt a bivalent-promoting mechanism and reveal cryptic homologies, probably due to the presence of $P h$-like gene (Pairing homologous) such as the $P h l$ gene which occur in Triticum aestivum (Feldman and Avivi, 1988).

It is interesting to note that molecular studies (Moore et al., 1995) are in agreement with our cytogenetic findings and support the cryptic allotetraploid nature of maize.

\section{Molecular cytogenetic analysis of species and hybrids}

Genomic in situ hybridization (GISH) allows chromosomes from different parents or ancestors to be distinguished by means of differential hybridization of entire genomic probes (Bennett, 1995). Moreover, GISH has proved to be a very useful technique to demonstrate affinities among putative ancestors of $Z$. mays ssp. mays (Poggio et al., 1998, 1999a,b).

By applying GISH to Z. luxurians we demonstrated that the DAPI positive bands located in all telomeric regions of this species did not hybridize either with $Z$. perennis or Z. diploperennis genomic probes, whereas the rest of the chomosome regions showed hybridization signals. Z. luxurians therefore has a repetitive sequence that can be used to identify its chromosomes by fluorescent staining techniques (Poggio et al., 1999b).

We studied metaphase I in Z. luxurians $\mathrm{x} Z$. perennis $(2 \mathrm{n}=30)$ hybrids, which (similar to the previous crosses) had a metaphase 1 chromosome complement of 5 trivalents +5 bivalents +5 univalents as the most frequent configuration (Figure 1). When fluorescent techniques were employed, the distinctive telomeres of Z. luxurians were found on the univalents but not on the bivalents (Poggio et al., 1999b), findings which show that the formation of bivalents and univalents is not a random event. In Z. luxurians $\mathrm{x} Z$. perennis hybrids, bivalents result from pairing between chromosomes from the two genomes of Z.peren$n i s$ with the exclusion of the corresponding chromosomes from the Z. luxurians genome (Figure 1). In this hybrid, bivalents from $Z$. perennis tend to be spatially separated and are very often observed forming an independent group of 5 bivalents. The GISH experiments confirm that genomes belonging to different species tend to occupy different domains in the nucleus, supporting the hypothesis that there is a tendency to genome separation.

Repetitive sequences can be used as species makers to differentiate chromosomes from different species. To gain further insight into the nature of these sequences, we present below the results of further hybridization experiments applied to different Zea species which we hope will contribute to resolving many of the questions relating to molecular affinities between our proposed genomes for the genus Zea.

\section{MATERIAL AND METHODS}

\section{Plant material}

The source of material for cytogenetical analysis was: Zea perennis: Mexico, Jalisco, Ciudad Guzmán, Leg. Dra. Prywer, cultivated at the "Instituto Fitotécnico Santa Catalina (IFSC)" since 1962; Z. diploperennis: Mexico, Jalisco, Sierra de Mananthan Occidental, $2 \mathrm{~km}$ from Las Joyas, Leg. Rafael Guzman and M.A. Guzman (No. 1120, Nov. 1980); Z. luxurians: Guadalajara, Mexico Cult. No. 2228 (IFSC) and Z. mays ssp. mays line (Santa Catalina 2), maintained in the greenhouse of the IFSC.

Materials analyzed by the Southern blotting method were: Zea mays ssp. mays (SC2 line and accession 13043, IFSC) and the following accessions provided by CIMMYT 
(Centro International de Mejoramiento de Maíz y Trigo): Z. luxurians (9478), Tripsacum dactyloides, Z. diploperennis (933) and Z. perennis (8837).

$$
\text { Cytological analysis }
$$

For fluorescent in situ hybridization seeds were placed on Petri dishes on wet filter paper. Root tips were pretreated in $0.02 \mathrm{M}$ 8-hydroxyquinoline and fixed in 3:1 (absolute alcohol:acetic acid). Fixed roots were washed in $0.01 \mathrm{M}$ citric acid-sodium citrate buffer, $\mathrm{pH} 4.6$, to remove fixative, transferred to an enzyme solution containing $2 \mathrm{ml}$ of $2 \%$ cellulase (Onozuka R10) and $20 \%$ liquid pectinase (Sigma). The softened material was again washed in the above buffer solution. Finally, chromosomes were squashed onto slides in a drop of $45 \%$ acetic acid. Preparations showing well-spread cells were selected by phase contrast light microscopy. After removing the coverslips (by freezing the slides) the slides were air-dried.

\section{Molecular analysis}

\section{DNA probes}

The following probes were used for in situ hybridization: 1) a 180-bp repeat of the maize heterochromatic knob (Dennis and Peacock, 1984 - kindly provided by CSIRO Plant Industries), 2) the pTa 71 plasmid containing the 18S-5.8S-25S ribosomal sequences from Triticum aestivum (Gerlach and Bedbrook, 1979) and 3) genomic DNA isolated from adult leaves of the three species of the Luxuriantes Section (after Maniatis et al. (1982), with minor modifications). All probes were labeled by random priming with digoxigenin 11-dUTP (Boehringer, Mannheim, Germany) or by nick translation with biotin 14-dUTP (Bionick Labeling System, Gibco BRL).

\section{Southern-blot hybridization}

For this technique, $10 \mu \mathrm{g}$ of DNA from each of the species listed in plant material was digested with HindIII, EcoRI, RsaI and PstI endonucleases. Fragments were electrophoretically separated on $0.8 \%$ agarose gels, blotted on nylon membranes and hybridized with digoxigenin labeled knob-specific probe (Maniatis et al., 1982), with post-hybridization washes under high stringency conditions. Immunodetection of probes was carried out according to the manufacturer's instructions (Boehringer-Mannheim).

\section{Fluorescent in situ hybridization}

The technique, slightly modified, of Cuadrado and Jouve (1995) was used. Slide preparations were incubated in $100 \mu \mathrm{g} / \mathrm{ml}$ DNAse-free RNAse in $2 x$ SSC for $1 \mathrm{~h}$ at $37^{\circ} \mathrm{C}$ in a humidified chamber and washed three times in $2 x \mathrm{SSC}$ at room temperature for $5 \mathrm{~min}$. The slides were post-fixed in freshly prepared $4 \%(\mathrm{w} / \mathrm{v})$ paraformaldehyde in water for $10 \mathrm{~min}$, washed in 2x SSC for $15 \mathrm{~min}$, dehydrated in a graded ethanol series and air dried. The hybridization mixture consisted of $50 \%(\mathrm{v} / \mathrm{v})$ deionized formamide, $10 \%(\mathrm{w} /$ v) dextran sulphate, $0.1 \%(\mathrm{w} / \mathrm{v}) \mathrm{SDS}$ and $0.3 \mathrm{mg} / \mathrm{ml}$ of salmon sperm DNA in 2x SSC, followed by adding $100 \mathrm{ng}$ of labeled probe to $30 \mu \mathrm{l}$ of hybridization mixture for each slide. In some cases, a blocking procedure (Bennett, 1995) was applied by adding unlabeled DNA from a different species and labeled total DNA probe from the same species in a proportion of 10:1, respectively. The hybridization mixture was denatured for $15 \mathrm{~min}$ at $75^{\circ} \mathrm{C}$, loaded onto the slide preparation and covered with a plastic coverslip. The slides were placed on a thermocycler at $75^{\circ} \mathrm{C}$ for $7 \mathrm{~min}$ (denaturation), $10 \mathrm{~min}$ at $45^{\circ} \mathrm{C}$ and $10 \mathrm{~min}$ at $38^{\circ} \mathrm{C}$. The slides were then incubated overnight at $37^{\circ} \mathrm{C}$ for hybridization.

Following hybridization, coverslips were carefully floated off by placing the slides in $2 x \mathrm{SSC}$ at $42^{\circ} \mathrm{C}$ for 3 min and then given a stringent wash in $20 \%$ formamide in $0.1 \mathrm{x} \mathrm{SSC}$ at $42^{\circ} \mathrm{C}$ for $10 \mathrm{~min}$. The slides were washed in $0.1 \mathrm{x} \mathrm{SSC}$ at $42^{\circ} \mathrm{C}$ for $5 \mathrm{~min} ; 2 \mathrm{x} \mathrm{SSC}$ for $5 \mathrm{~min}$ at $42^{\circ} \mathrm{C}$ and transferred to detection buffer (4x SSC, $0.2 \%$ (v/v) Tween 20) for $5 \mathrm{~min}$ at $42^{\circ} \mathrm{C}$ and $1 \mathrm{~h}$ at room temperature.

To detect digoxigenin-labeled probes, slides were treated with sheep antidigoxigenin FITC (fluorescein isothyocyanate) (green fluorescence) while for biotin-labeled probes they were treated with streptavidine-Cy3 conjugate (red fluorescence) or avidin Texas red. Slides were then treated with $2.5 \%(\mathrm{w} / \mathrm{v}) \mathrm{BSA}$ in detection buffer and incubated in a $1 / 40$ solution of the corresponding antibody in detection buffer containing $2.5 \% \mathrm{BSA}$ for $1 \mathrm{~h}$ at $37^{\circ} \mathrm{C}$ and washed 3 times in detection buffer for $10 \mathrm{~min}$ at room temperature.

Slides were counterstained with $1 \mu \mathrm{g} / \mathrm{ml}$ of 4' 6 diamidino-2-phenylindole (DAPI) in McIlvaine's citrate buffer, $\mathrm{pH}=7$, for $10 \mathrm{~min}$ at room temperature and then mounted in anti-fade solution. Slides were examined with a Carl Zeiss Axiophot epifluorescence microscope with appropriate Carl Zeiss filters. Photographs were taken using Fuji color super G400 color print film.

\section{RESULTS AND DISCUSSION}

We performed several molecular hybridization experiments to obtain information about the DNA sequences which form the DAPI-positive telomeric regions of $Z$. luxurians chromosomes but which are not present in the other members of the Zea section. These experiments are described below.

In situ hybridization using the knob sequence as a probe for Z. luxurians chromosomes

Terminal heterochromatic blocks of Z. luxurians (ZLTHB) look like those of $Z$. mays ssp. mays (maize) when 
observed using conventional C-banding techniques (Tito $e t$ $a l ., 1991$ ) or by DAPI counterstaining (Figure 2A, C, and D). We therefore decided to assess the homology of the ZL-THB with respect to the 180 -bp repeat (Dennis and Peacock, 1984) contained in maize heterochromatic knobs.

We performed an in situ hybridization experiment probing the knob sequence on mitotic chromosomes of $Z$. luxurians in which we observed that all DAPI-positive telomeric regions fluoresced (Figure 2B, D, F), indicating that the ZL-THB contain the 180 -bp repeat.

\section{Southern blot analysis using the knob sequence as a probe}

Dennis and Peacock (1984) reported that the knob sequence is also the major component of heterochromatic knobs in the annual grass teosinte (Z. mexicana, Mexican teosinte, and Z. luxurians, Guatemala teosinte), as well as in Z. diploperennis and Tripsacum. We confirmed these findings by Southern blot analysis and also demonstrated that $Z$. perennis (or at least the accessions we used) lacks the knob sequence.

\section{Genomic in situ hybridization of Z. luxurians chromosomes}

As previously mentioned, GISH experiments had demonstrated that ZL-THB regions are not present on either $Z$. perennis or $Z$. diploperennis chromosomes (Poggio et al., 1999b). The ZL-THB region should, therefore, be composed of more than one sequence, as the 180-bp repeat is not unique to Z. luxurians within its section, and in order to confirm this we performed other GISH experiments.

We carried out the hybridization procedure on mitotic chromosomes from $Z$. luxurians using labeled total genomic DNA from $Z$. luxurians as a probe, blocking with unlabeled DNA from Z. perennis (Figure $2 \mathrm{H}$ ) or Z. diploperennis (Figure $2 \mathrm{~J}$ ). In both cases strong fluorescence was only present on telomeric regions having the same position as DAPI-positive bands (Poggio et al., 1999b). Taking into account the observed homologies of the knob sequence to ZL-THB region this result was expected when using $Z$. perennis as a blocking agent. However, lack of fluorescence in the ZL-THB region should have been observed when GISH was performed using unlabeled DNA from $Z$. diploperennis, since this DNA should block the telomeric region because of its homology with the knob sequence. We have thus concluded that the ZL-THB region contains not only the 180-bp repeat, but also other tandem arrays unique to $Z$. luxurians. In fact, Figure $2 \mathrm{~J}$ (blocked with $Z$. diploperennis) shows that fluorescent regions seem to be smaller and subterminal in most chromosomes compared to Figure 2E (blocked with Z. perennis), suggesting that the unique $Z$. luxurians sequence is probably located at the subterminal position.
Southern blot analysis using the knob sequence as a probe on Z. luxurians cleaved DNA

Previous work has shown that some endonucleases (including HindIII, EcoRl, RsaI and PstI) cleave total genomic DNA from $Z$. luxurians in such a way that a ladder pattern can be visualized with ethidium bromide, but these endonucleases do not have recognition sites on the 180-bp repeat (Poggio et al., 1999c).

However, when we performed Southern blot analysis using DNA from $Z$. luxurians digested with these endonucleases and hybridized with knob probe, we surprisingly observed that the probe hybridized producing a ladder pattern. The fact that the endonucleases employed do not have recognition sites on the 180-bp repeat suggests that in $Z$. luxurians another sequence with target sites for these enzymes could be interspersed with the knob repeat and be organized with them in a tandem array. These arrays of "knobs plus other sequences with target sites for endonucleases" are located on the ZL-THB regions as was demonstrated by using GISH (Figure 2F).

$Z$. mays ssp. mays and $Z$. diploperennis showed on Southern blot analysis a unique band of homology when hybridized with knob probe. Indeed, Phelps and Birchley (1997) stated that the 180-bp repeat seems to be the only sequence present in heterochromatic knobs of $Z$. mays ssp. mays. However, Ananiev et al. (1998) found a Tr-1 tandem repeat array up to $70 \mathrm{~kb}$ in length interspersed with stretches of 180-bp tandem arrays. A similar type of array could be present in Z. luxurians. Nevertheless, the interspersed array would be a different sequence because of the different patterns observed among the Zea species that were cleaved with the endonucleases and hybridized with the knob probe in our Southern blot analysis.

These observations indicate an important differentiation between the species of the genus Zea. A more detailed study on the organization of tandem arrays will perhaps provide a clue to determine the evolutionary relationships between these species, research that is currently in progress in our laboratories.

\section{Genomic in situ hybridizations of Z. mays ssp. mays chromosomes}

The heterochromatic knobs from Zea mays ssp. mays correspond to DAPI-positive bands on mitotic chromosomes of maize (Sumner, 1990). The distribution, number and size of knobs vary among different lines of maize. The GISH experiment using Z. luxurians as a probe for maize chromosomes showed stronger fluorescence in the knob regions than in the rest of the chromosome (Figure 2L). The same cell re-hybridized with $Z$. diploperennis as a probe produced strong fluorescence on the whole chromosome, including the knob regions (Figure 2M). Similar results were observed in other cells (Figure 2Q) suggesting that the affinity between $Z$. diploperennis and Z. mays 

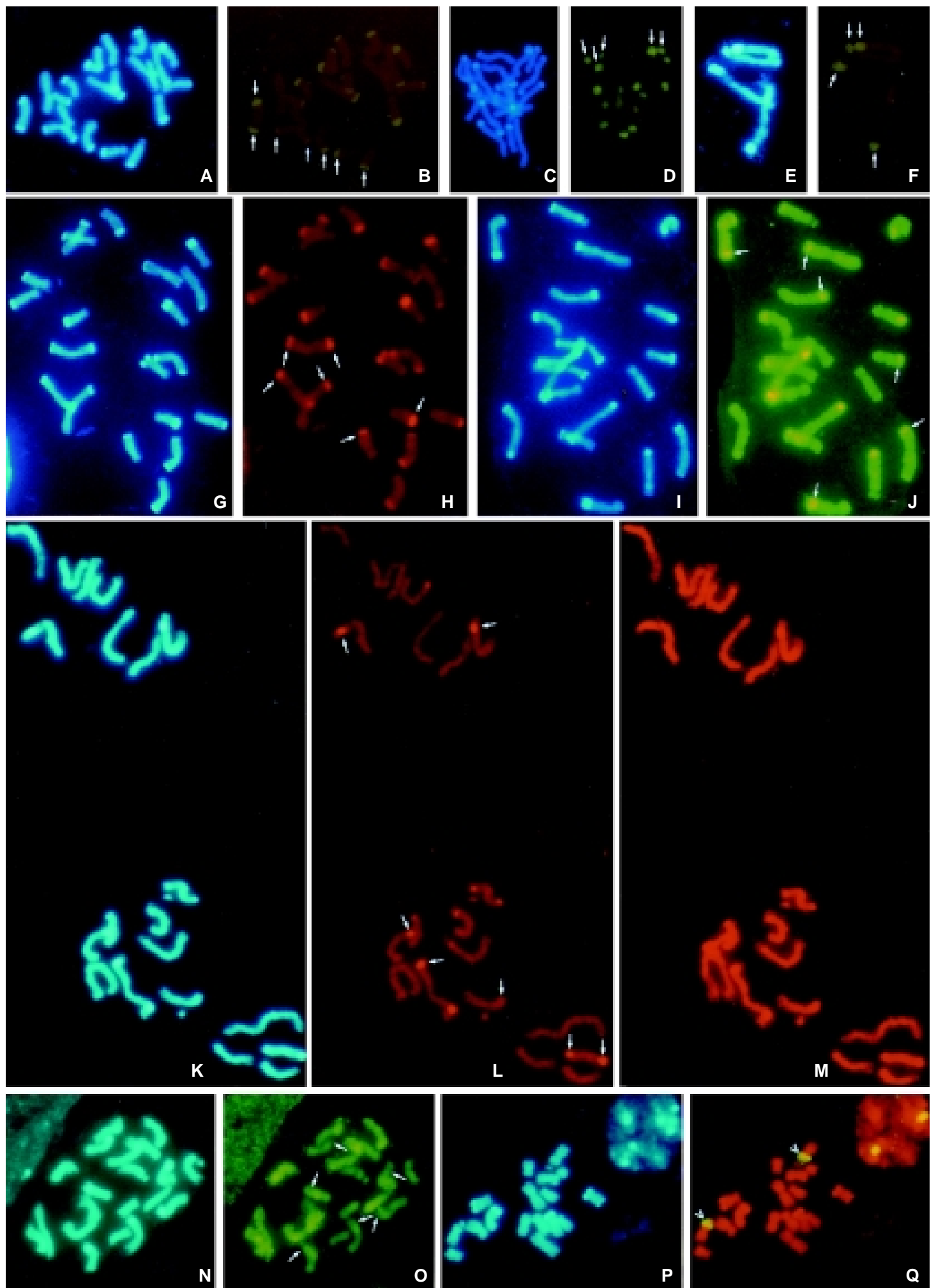

Figure 2 - A-J, Mitotic cells of Z. luxurians. A, Metaphase. C, Prophase. A, C, E, G, I, DAPI counterstaining. B, D, F, FISH using knob probe, detected with yellow green FITC (arrows indicate some of the telomeric regions with fluorescent regions coincident with DAPI + bands). E, Detail from four prophase chromosomes. H, GISH using DNA from Z. luxurians as a probe, detected with red Cy3, blocked with DNA from Z. perennis (arrows indicate some of the regions with strong fluorescence coincident with DAPI + bands). J, GISH using DNA from Z. luxurians as a probe detected with yellow green FITC, blocked with Z. diploperennis (arrows indicate some of the subtelomeric regions with fluorescence coincident with the DAPI + bands). K-Q, Mitotic cells from Zea mays ssp. mays. K, N, P, DAPI counterstaining. L, GISH using Z. luxurians DNA as a probe detected with avidin Texas red (arrows indicate some of the regions with strong fluorescence coincident with DAPI + bands). M, GISH using Z. diploperennis DNA as a probe detected with avidin Texas red. O, GISH using total DNA from Z. perennis as a probe detected with yellow green FITC (arrows show some of the regions of DAPI + bands exhibiting weaker fluorescence). Q, Double exposure photograph using total DNA from Z. diploperennis as a probe (red) and pTa 71 (yellow green, indicated with arrows). 
ssp. mays is greater than that between $Z$. luxurians and $Z$. mays ssp. mays.

When total DNA of Z. perennis was probed on Z. mays ssp. mays chromosomes the results were in agreement with Southern blot analysis. As Z. perennis lacks the 180-bp repeat, weaker fluorescence was observed in the regions corresponding to heterochromatic knobs (Figure 2O). Interestingly, GISH analysis also revealed that the Z. perennis probe does not hybridize uniformly with $Z$. mays ssp. mays chromosomes.

This pattern of labeling could indicate that more than one species may have been involved in the origin of maize and that rearrangements between genomes could have occurred later, an idea which is in agreement with the proposed cryptic alloploid nature of maize.

\section{ACKNOWLEDGMENTS}

The authors would like to thank Ovidio Nunez for helpful comments on the original manuscript and Nicolas Jouve and Angelines Cuadrado for their invaluable help regarding the use of the GISH procedure as well as for facilities offered during the visit of L. Poggio \& C.A. Naranjo to the Department of Cellular Biology and Genetics, Alcala University (Spain). This research was supported by grants from the Consejo Nacional de Investigaciones Cientifícas y Técnicas (CONICET), the Universidad de Buenos Aires, the Universidad Nacional de La Plata, Argentina and the Agencia de Promoción Científica y Tecnológica. L. Poggio, V. Confalonieri and C.A. Naranjo belong to the Argentine Research Council (CONICET). G. Gonzalez was supported by scholarships from Comisión de Investigaciones Científicas de la Prov. de Buenos Aires (CIC).

\section{REFERENCES}

Ananiev, E.V., Phillips, R.L. and Rines, H.W. (1998). A knob-associated tandem repeat in maize capable of forming fold-back DNA segments: are chromosome knobs megatransposons? Proc. Natl. Acad. Sci. USA 95: 10785-10790.

Bennett, M.D. (1983). The spatial distribution of chromosomes. In: Kew Chromosome Conference II (Brandham, P.E. and Bennett, M.D., eds.). Allen \& Unwin, London, pp. 71-79.

Bennett, M.D. (1984). The genome, the natural karyotype and biosystematics. In: Plant Biosistematics (Grant, W.F., ed.). Academic Press, New York, pp. 41-66.

Bennett, M.D. (1995). The development and use of genomic in situ hybridization (GISH) as a new tool in plant biosystematics. In: Kew Chromosome Conference IV (Brandham, P.E. and Bennett, M.D., eds.). Royal Botanic Gardens, Kew, pp. 167-183.

Cuadrado, A. and Jouve, N. (1995). Fluorescent in situ hybridization and C-banding analyses of highly repetitive DNA sequence in the heterochromatin of rye (Secale montanum Guss.) and wheat incorporating S. montanum chromosome segments. Genome 38: 795-802.

Dennis, E.S. and Peacock, W.J. (1984). Knob heterochromatin homology in maize and its relatives. J. Mol. Evol. 20:341-350.

Doebley, J.F. and IItis, H.H. (1980). Taxonomy of Zea. 1. Subgeneric classification with key to taxa. Am. J. Bot. 67: 982-993.

Feldman, M. and Avivi, L. (1988). Genetic control of bivalent pairing in common wheat. The mode of $P h 1$ action. In: Kew Chromosome Conference III (Brandham, P.E., ed.). Royal Botanic Gardens, Kew, pp. 269-279.
Gerlach, W.L. and Bedbrock, J.R. (1979). Cloning and characterization of ribosomal RNA genes from wheat and barley. Nucleic Acids Res. 7: 1869-1885.

Iltis, H.H. and Doebley, J.F. (1980). Taxonomy of Zea (Gramineae). 2. Subspecific categories in the Zea mays complex and a generic synopsis. Am. J. Bot. 67: 994-1004

Jackson, R.C. and Murray, B.G. (1983). Colchicine-induced quadrivalent formation in Helianthus: evidence of ancient polyploidy. Theor. Appl. Genet. 64: 219-222.

Jouve, N., Soler, C. and Saing, G. (1977). Cytoplasmic influence on the meiosis of 6x-Triticale. Z. Pflanzenzuecht. 78: 124-134.

Longley, A.E. (1924). Chromosomes of maize and maize relatives. J. Agric. Res. 28: 673-681.

Maniatis, T., Fritsch, E.F. and Sabrook, J. (1982). Molecular Cloning: A Laboratory Manual. Cold Spring Harbor Laboratory, Cold Spring Harbor, New York.

McClintock, B. (1933). The association of non-homologous parts of chromosomes in the midprophase of meiosis in Zea mays. Z. Zellforsch. Mikrosk. Anat. 19: 191-237.

Molina, M.C. and Naranjo, C.A. (1987). Cytogenetic studies in the genus Zea. 1. Evidence for five as the basic chromosome number. Theor. Appl. Genet. 73: 542-550.

Moore, G., Devos, K.M., Wang, Z. and Gale, M.D. (1995). Cereal genome evolution. Grasses, line up and form a circle. Curr. Biol. 5: 737-739.

Naranjo, C.A., Molina, M.C. and Poggio, L. (1990). Evidencias de un número básico $\mathrm{X}=5$ en el género Zea y su importancia en estudios del origen del maíz. Acad. Nac. Cienc. Ex. Fis. Nat. Buenos Aires, Monografía 5: 43-53.

Naranjo, C.A., Poggio, L., Molina, M. and Bernatené, E. (1994). Increase in multivalent frequency in $\mathrm{F} 1$ hybrids of Zea diploperennis $\mathrm{x} Z$. perennis by colchicine treatment. Hereditas 120: 241-244.

Phelps, T. and Birchley, J.A. (1997). Molecular organization and size estimation of chromosome knobs in maize. Maize Genetics Conference Abstracts, 39

Poggio, L. and Naranjo, C.A. (1995). Cytogenetic analysis of hybrids in Zea: evolutionary consideration. 12th International Chromosome Conference, Madrid, Spain. Chromosome Res. 3 (Suppl. 1): 81.

Poggio, L., Molina, M.C. and Naranjo, C.A. (1990). Cytogenetic studies in the genus Zea. 2. Colchicine-induced multivalents. Theor. Appl. Genet. 79: 461-464

Poggio, L., Rosato, M. and Naranjo, C.A. (1997). Meiotic behavior in alloplasmic lines of Zea mays spp. mays. Genome 40: 723-729.

Poggio, L., Confalonieri, V., Comas, C., Gonzalez, G. and Naranjo, C.A. (1998). Genomic affinities of Zea luxurians, Z. diploperennis and $Z$. perennis: meiotic behaviour of their $\mathrm{F} 1$ hybrids and (GISH). In 13th International Chromosome Conference Ancona Italia (Olmo, E., ed.). Cytogenet. Cell Genet. (Abstracts) 81: 134.

Poggio, L., Confalonieri, V., Comas, C., Cuadrado, A., Jouve, N. and Naranjo, C.A. (1999a). Genomic in situ hybridization (GISH) of Tripsacum dactyloides and Zea mays ssp. mays with B-chromosomes. Genome 42: 687-691.

Poggio, L., Confalonieri, V., Comas, C., Gonzalez, G. and Naranjo, C.A. (1999b). Genomic affinities of Zea luxurians, Z. diploperennis and Z. perennis: meiotic behaviour of their F1 hybrids and genomic in situ hybridization (GISH). Genome 42: 993-1000.

Poggio, L., Confalonieri, V., Comas, C., Gonzalez, G. and Naranjo, C.A. (1999c). Análisis de secuencias repetidas utilizadas como marcadores citológicos: Relaciones evolutivas en Zea. Actas XXIX Congreso Argentino de Genética, Septiembre 1999, pp. 227.

Sumner, A.T. (1990). Chromosome Banding. Unwin Hyman, London, pp. 434.

Ting, Y.C. (1985). Meiosis and fertility of anther culture-derived maize plant. Maydica 30: 161-169.

Tito, C., Poggio, L. and Naranjo, C.A. (1991). Cytogenetics studies in the genus: DNA content and heterochromatin in species and hybrids. Theor. Appl. Genet. 83: 58-64.

Vijendra Das, L.D. (1970). Chromosome associations in diploid and autotetraploid Zea mays L. Cytologia 35: 259-261. 
